\title{
ANALYTIC MAPS BETWEEN RIEMANN SURFACES ${ }^{1}$
}

\author{
DALE H. HUSEMOLLER ${ }^{2}$
}

We use the term Riemann surface to mean a pair consisting of a Riemann surface in the usual sense (see [2, p. 114]) and a base point. The only analytic maps considered are those which carry the base point on the base point, and all (open) subsurfaces have for base point the base point of the ambient space. Except in \$1, we consider only Riemann surfaces with the unit disc $U$ as their universal covering surface. Zero is the base point of $U$.

Let $A(X, Y)$ denote the set of all analytic maps from the Riemann surface $X$ to the Riemann surface $Y$. In the first three sections we develop the notion of approximation of maps in $A(X, Y)$ by maps in $A(W, Y)$ where $W$ is a subsurface of $X$. This is done by considering sequences of subsurfaces $W_{n}$ of $X$ which converge to $X$, in a sense made precise in $\S 2$, and functions $f_{n} \in A\left(W_{n}, Y\right)$ such that for the liftings $f_{n}^{0}, f^{0}$ to the universal covering surface, we have $f_{n}^{0} \rightarrow f^{0}$ in the topology of compact convergence. Then we say that $f_{n}$ approximates $f$. We prove that $f$ can always be approximated by $f_{n}$ defined on relatively compact $W_{n}$. Moreover, for all sequences $f_{n} \in A\left(W_{n}, Y\right)$ there exists a subsequence approximating a map in $A(X, Y)$. In the last section we use this result to prove that $A(X, Y)$ is a compact space.

1. Simply embedded subsurfaces. If $W$ is a subsurface of a Riemann surface $X$, then we denote by $i$ the natural inclusion mapping of $W$ into $X$. Let $\left(X^{0}, \pi\right)$ (or $\left(X^{0}, \pi_{X}\right)$ ) denote the universal cover of $X$, see [2, p. 37]. If $f: X \rightarrow Y$ is an analytic map, then there exists a unique analytic map $f^{0}: X^{0} \rightarrow Y^{0}$ such that $\pi_{Y} \circ f^{0}=f \circ \pi_{X}$, and $f$ induces a group homomorphism $f_{*}: \pi_{1}(X) \rightarrow \pi_{1}(Y)$.

Definition 1. A subsurface $W$ of $X$ is simply embedded in $X$ if the induced homomorphism $i_{*}: \pi_{1}(W) \rightarrow \pi_{1}(X)$ is injective.

In other words, $W$ is simply embedded in $X$ if and only if for each loop in $W$ which is homotopic to zero when viewed in $X$ is homotopic to zero when viewed in $W$. All homotopies are with fixed base point. If $i_{*}: \pi_{1}(W) \rightarrow \pi_{1}(X)$ is injective for one base point in $W$, then $i_{*}$ is injective for all base points in $W$.

Let $W$ be a subsurface of $X$ and let $u$ be a loop in $W$. Let $u^{W}$

Received by the editors November 7, 1960 and, in revised form, January 18, 1961 and March 20, 1961.

${ }^{1}$ Some of the material in this paper can be found in the author's thesis presented in partial fulfillment of the requirements for a $\mathrm{Ph}$.D. at Harvard, 1959.

${ }^{2}$ This work was partially supported by Air Force contract AF 181600-1461. 
(resp. $u^{x}$ ) be the unique lifting of $u$ to a path in $W^{0}$ (resp. $X^{0}$ ) beginning at the base point. The loop $u$ is homotopic to zero in $W$ (resp. $X$ ) if and only if $u^{W}$ (resp. $\left.u^{X}\right)$ is a loop in $W^{0}\left(\operatorname{resp} . X^{0}\right)$. Since $u^{X}=i^{0} \circ u^{W}$, we have the following result.

Proposition 1. Let $W$ be a subsurface of $X$ with natural injection map $i: W \rightarrow X$. The subsurface $W$ is simply embedded in $X$ if and only if $i^{0}: W^{0} \rightarrow X^{0}$ is injective.

Using Proposition 1 or a direct argument, we can give new, simple proofs of Satz 1 and Satz 2 in H. Behnke and K. Stein [3, pp. 435$436]$ in the next proposition. Behnke and Stein used properties of triangulations of Riemann surfaces in their proofs.

Proposition 2. Let $X$ be an open Riemann surface, and let $K$ be a compact subset of $X$. Then there exists a relatively compact, simple embedded subsurface $W$ of $X$ such that $K \subset W$.

Proof. The universal covering surface $X^{0}$ is conformally equivalent to either the plane $C$ or the unit disc $U$. In the case of the plane let $X(r)(0<r)$ be the square centered at $0 \in C$ with sides of length $2 r$, and in the case of the unit disc let $X(r)(0<r<1)$ be the set of $z \in U,|z|<r$. There exists an $r$ with $\pi(X(r)) \supset K$. The subsurface $W=\pi(X(r))$ has $\pi \mid X(r): X(r) \rightarrow W$ as its universal covering. Moreover, we see that the subsurface $W$ is simply embedded in $X$ by using Proposition 1 and the injection $X(r) \rightarrow X^{0}$ or by the fact that every loop in $X^{0}$ is homotopic to a loop in $X(r)$ by radial shrinking. q.e.d.

2. Approximating sequences of subsurfaces. Each sequence of subsurfaces $\left\{W_{n}\right\}$ of $X$ that we consider, henceforth, has the following properties: For all $n, W_{n} \subset W_{n+1}, W_{n}$ is simply embedded in $X$, and for all compact subsets $K$ of $X$ there exists $q$ such that $K \subset W_{q}$. By Proposition 2 we see that there exists an exhaustion $\left\{W_{n}\right\}$ which is a sequence of subsurfaces. Also, $W_{n}=X$ for each $n$ is a sequence of subsurfaces.

For a sequence of subsurfaces $\left\{W_{n}\right\}$ of $X$ we use the following notations. Let $(U, \pi, X)$ (resp. $\left(U, \pi_{n}, W_{n}\right)$ ) denote the universal covers of $X$ (resp. $W_{n}$ ), and let $G(\pi)$ (resp. $G\left(\pi_{n}\right)$ ) be the group of covering transformations of $U \rightarrow U$ of the covering $(U, \pi, X)$ (resp. $\left.\left(U, \pi_{n}, W_{n}\right)\right)$. The natural inclusion map $i_{n}: W_{n} \rightarrow X$ determines a unique "lifting" $i_{n}^{0}: U \rightarrow U$ and induces a group homomorphism $\theta_{n}: G\left(\pi_{n}\right) \rightarrow G(\pi)$. The natural inclusion map $i_{q, n}: W_{n} \rightarrow W_{q}(n \leqq q)$ determines a unique "lifting" $i_{q, n}^{0}: U \rightarrow U$ and induces a group homomorphism $\theta_{q, n}: G\left(\pi_{n}\right) \rightarrow G\left(\pi_{q}\right)$. We have the relations $i_{q}^{0} \circ i_{q, n}^{0}=i_{n}^{0}$ and $\theta_{q} \circ \theta_{q, n}=\theta_{n}$ for all $n, q$ with $n \leqq q$. For all $S \in G\left(\pi_{n}\right), \theta_{n}(S) \circ i_{n}^{0}=i_{n}^{0} \circ S$ 
and $\theta_{q, n}(S) \circ i_{q, n}^{0}=i_{q, n}^{0} \circ S(n \leqq q)$. We assume that $\pi_{n}$ was chosen relative to $\pi$ such that $i_{n}^{0 \prime}(0)>0$ for all $n$.

Definition 2. A sequence of subsurfaces $\left\{W_{n}\right\}$ approximates $X$ if $i_{n}^{0}$ converges to the identity in $A(U)$.

For example $W_{n}=X($ all $n$ ) is an example of a sequence of subsurfaces which approximate $X$. In the next proposition we derive a method which will enable us to find nontrivial approximations.

Proposition 3. Let $\left\{W_{n}\right\}$ be a sequence of subsurfaces of $X$. If $i_{n}^{0}$ converges to $h$ in $A(U)$, then $h(z)=z$, all $z \in U$.

Proof. The function $h$ is surjective. From the relations $i_{q}^{0} \circ i_{q, n}^{0}=i_{n}^{0}$ and $\theta_{q} \circ \theta_{q, n}=\theta_{n}$ for $n \leqq q$, we have $\operatorname{im}\left(i_{n}^{0}\right) \subset \operatorname{im}\left(i_{q}^{0}\right)$ and $\operatorname{im}\left(\theta_{n}\right) \subset \operatorname{im}\left(\theta_{q}\right)$. Let $u \in U$. There exists $m$ such that $\pi(u) \in W_{q}(m \leqq q)$, and there exists $u_{q}^{\prime} \in U$ such that $\pi_{q}\left(u_{q}^{\prime}\right)=\pi(u)(m \leqq q)$. There exists $T \in G(\pi)$ such that $T(u) \in \operatorname{im}\left(i_{m}^{0}\right) \subset \operatorname{im}\left(i_{q}^{0}\right) \quad(m \leqq q)$. Let $n \geqq m$ such that $T=\theta_{n}\left(T_{n}\right), T_{n} \in G\left(\pi_{n}\right)$. From the relation $T \circ i_{n}^{0}=i_{n}^{0} \circ T_{n}$ applied to $T_{n}^{-1}\left(u^{\prime}\right)$ where $i_{n}^{0}\left(u^{\prime}\right)=T(u)$, we get $\left.T\left(i_{n}^{0}\left(T^{-1}\left(u^{\prime}\right)\right)\right)=i_{n}^{0}\left(T_{n}\left(T_{n}^{-1}\right) u^{\prime}\right)\right)$ ) $=i_{n}^{0}\left(u^{\prime}\right)=T(u)$ and $u=i_{n}^{0}\left(T^{-1}\left(u^{\prime}\right)\right)$. Therefore, $u \in \operatorname{im}\left(i_{q}^{0}\right)$ for all $q \geqq n$.

Let $u_{n} \in U$ such that $i_{n}^{0}\left(u_{n}\right)=u$, and let $u_{q}=i_{q, n}^{0}\left(u_{n}\right)$ for all $q \geqq n$. Then $i_{q}^{0}\left(u_{q}\right)=u$ for all $q \geqq n$, and by Schwarz's lemma applied to the function $i_{q, n}^{0}$, we have $\left|u_{q}\right| \leqq\left|u_{n}\right|$ for all $q \geqq n$. Since the set of $z \in U$, $|z| \leqq\left|u_{n}\right|$ is compact, there exists a subsequence $u_{q(i)} \rightarrow u^{*}$ in $U$. Then $u=i_{q(i)}^{0}\left(u_{q(i)}\right)$ converges to $h\left(u^{*}\right)$ by the iterated limits theorem [4, Theorem 2, p. 20]. Therefore, $u \in \operatorname{im}(h)$, and $h$ is surjective.

Since a limit of injective functions is injective or constant by Hurwitz's theorem, the function $h$ is injective of constant. The function $h$ is not constant because it is surjective, and therefore, $h$ is a bijection.

Since $h(0)=0$, we have $h(z)=e^{i \theta} z$, and since $i_{n}^{-0}(0) \rightarrow h^{\prime}(0)=e^{i \theta}$ and $i_{n}^{0 \prime}(0) \geqq 0$, we have $e^{i \theta}=1$ and $h(z)=z$. q.e.d.

Corollary 1. Let $\left\{W_{n}\right\}$ be a sequence of subsurfaces of $X$. Then there exists a subsequence $\left\{W_{n(p)}\right\}$ approximating $X$.

Proof. Since $A(U)$ is compact, there exists a subsequence $i_{n(p)}^{0}$ of $i_{n}^{0}$ which converges to $h$ in $A(U)$. By Proposition $3, h$ is the identity and $\left\{W_{n(p)}\right\}$ approximates $X$. q.e.d.

Corollary 2. Let $\left\{W_{n}\right\}$ approximate $X$. Let $T \in G(\pi)$, and let $T_{q} \in G\left(\pi_{q}\right)(n \leqq q)$ such that $\theta_{q}\left(T_{q}\right)=T$. Then for all convergent subsequences $T_{q(k)}$ of $T_{q}$, we have $T_{q(k)} \rightarrow T$ in $A(U)$.

Proof. We have $T \circ i_{q(k)}^{0}=i_{q(k)}^{0} \circ T_{q(k)}$ for all $k$. Moreover, by the iterated limits theorem [4, Proposition 10, p. 22] we have $\lim _{k}\left(i_{q(\mathbf{k})}^{0} \circ T_{q(k)}\right)=\left(\lim _{k} i_{q(\mathbf{k})}^{0}\right) \circ\left(\lim _{k} T_{q(k)}\right)$. Therefore, we have 
$T=T \circ\left(\lim _{k} i_{q(\mathbf{k})}^{0}\right)=\lim _{k}\left(i_{q(k)}^{0} \circ T_{q(k)}\right)=\left(\lim _{k} i_{q(k)}^{0}\right) \circ\left(\lim _{k} T_{q(k)}\right)$ $=$ (identity) $\circ\left(\lim _{k} T_{q(k)}\right)$ and $T_{q(k)} \rightarrow T$. q.e.d.

By the compactness of $A(U)$, there always exists a convergent subsequence $T_{q(k)}$ of $T_{q}$.

Corollary 3. Let $\left\{W_{n}\right\}$ approximate $X$, and let $f \in A(X, Y)$. Then in the space $A(U), f^{0} \circ i_{n}^{0} \rightarrow f^{0}$.

Proof. We compute $\lim _{n}\left(f^{0} \circ i_{n}^{0}\right)=f^{0} \circ\left(\lim _{n} i_{n}^{0}\right)=f^{0} \circ$ (identity) $=f^{0}$. q.e.d.

3. Approximation by mappings defined on subsurfaces. If $f \in A(X, Y)$, then $f$ induces a homomorphism, denoted $f_{*}$, of $G\left(\pi_{\mathbf{X}}\right)$ into $G\left(\pi_{Y}\right)$. Now we are in a position to formulate the notion of approximation of an analytic map by maps defined on subsurfaces.

Definition 3. Let $\left\{W_{n}\right\}$ be a sequence of subsurfaces approximating $X$. A sequence of analytic maps $f_{n} \in A\left(W_{n}, Y\right)$ approximates $f \in A(X, Y)$ if $\lim _{n} f_{n}^{0}=f^{0}$ and $f_{*} \circ \theta_{n}=f_{n *}$ for all $n>N$.

There are two main results on approximation of maps. The first theorem says that every map can be approximated by maps defined of a sequence of subsurfaces which forms an exhaustion.

THEOREM 1. Let $\left\{W_{n}\right\}$ be a sequence of surfaces which approximates and exhausts $X$. Let $f \in A(X, Y)$. Then $f$ can be approximated by $f_{n} \in A\left(W_{n}, Y\right)$. In fact, we can take $f_{n}=f \mid W_{n}$.

Proof. Let $f_{n}=f \mid W_{n}$. Then $f_{n}^{0}=f^{0} \circ i_{n}^{0}$ and $\lim _{n} f_{n}^{0}=f^{0}$ by Corollary 3, Proposition 3. Moreover, from the definition of $f_{n}$, we have $f_{*} \circ \theta_{n}=f_{n * .}$ q.e.d.

The next theorem enables us to construct functions on arbitrary surfaces when they exist on subsurfaces. That is, for a coherent sequence of functions defined on subsurfaces of $X$ into another surface $Y$, there exists a subsequence which approximates a function on the whole surface.

THEOREM 2. Let $\left\{W_{n}\right\}$ be a sequence approximating $X$, and let $f_{n} \in A\left(W_{n}, Y\right)$ such that for $n \leqq q, f_{n *}=f_{q_{*}} \circ \theta_{q, n}$. Then there exists $n(p)$ and $f \in A(X, Y)$ such that $f_{n(p)}$ approximates $f$.

Proof. Since $A(U)$ is compact, there exists a subsequence $n(p)$ such that $f_{n(p)}^{0}$ converges to $g, g \in A(U)$. We wish to show $g=f^{0}$ where $f \in A(X, Y)$, or in other words, to show that there exists a homomorphism $\theta: G\left(\pi_{X}\right) \rightarrow G\left(\pi_{Y}\right)$ such that for all $T \in G\left(\pi_{X}\right), g \circ T=\theta(T) \circ g$.

Let $\theta$ have the defining property that $\theta \mid \operatorname{im}\left(\theta_{n}\right)=f_{n *} \circ \theta_{n}^{-1}$. This property is consistent for all $n$ by the coherence relation $f_{n *}=f_{q_{*}} \circ \theta_{Q, n}$ for $n \leqq q$.

Let $T \in G\left(\pi_{X}\right)$, and let $T=\theta_{n(p)}\left(T_{n(p)}\right)$ where $T \in \operatorname{im}\left(\theta_{n(p)}\right)$ for $p \geqq s$. 
By Corollary 2, Proposition 3, there exists a subsequence $m(p)$ of $n(p)$ such that $T_{m(p)} \rightarrow T$ in $A(U)$. Then for all $p$, we have $f_{m(p)}^{0} \circ T_{m(p)}$ $=\theta_{m(p)}\left(T_{m(p)}\right) \circ f_{m(p)}^{0}=\theta(T) \circ f_{m(p)}^{0}$. In the limit as $p \rightarrow+\infty$, using the iterated limits theorem [4, Proposition 10, p. 22], we get $g \circ T$ $=\theta(T) \circ g$ and $g=f^{0}$ for some $f \in A(X, Y)$. Moreover, $f_{n(p)}$ approximates $f$. q.e.d.

In the next section we derive an application of this result. $\mathrm{H}$. Behnke and K. Stein [3] have an analogue of our Theorem 1 for $Y=C$.

4. Topological properties of $A(X, Y)$. Let $X$ and $Y$ be two Riemann surfaces. On the set $A(X, Y)$ we have the topology of compact convergence, i.e., uniform convergence on compact sets. This topology is equivalent to the local uniform topology, i.e., uniform convergence on relatively compact coordinate discs.

In the special case $X=Y=U, A(U, U)$ (or $A(U)$ ) is the set of analytic functions $f$ on $U$ such that $|f(z)|<1$ for all $|z|<1$ and $f(0)=0$. The theory of normal families, see [1], says, in effect, that $A(U)$ is a compact separated space. We wish to extend this result to $A(X, Y)$ using the universal covering surfaces.

THEOREM 3. Let $X$ and $Y$ be two Riemann surfaces. The function $f \rightarrow f^{0}$ of $A(X, Y) \rightarrow A(U)$ is a homeomorphism of $A(X, Y)$ on a closed subset $A(X, Y)^{0}$ of $A(U)$. In particular, the space $A(X, Y)$ is compact.

Proof. The function $f \rightarrow f^{0}$ of $A(X, Y) \rightarrow A(X, Y)^{0} \subset A(U)$ is a bijection and the inverse function $L: A(X, Y)^{0} \rightarrow A(X, Y)$ is defined.

First we prove that $A(X, Y)^{0}$ is a closed subset of $A(X, Y)$. Let $f_{n}^{0} \in A(X, Y)^{0}$ such that $f_{n}^{0} \rightarrow g$ in $A(U)$. By Theorem 2 applied to the case $W_{n}=X$ for each $n$, there exists $f \in A(X, Y)$ and a subsequence $f_{n(p)}^{0} \rightarrow f^{0}$. Therefore, $f_{n}^{0} \rightarrow f^{0}=g$ and $A(X, Y)^{0}$ is a closed and compact subset of $A(U)$.

Let $f_{n}^{0} \rightarrow f^{0}$ in $A(X, Y)^{0}$. Then $f_{n} \circ \pi_{X}=\pi_{Y} \circ f_{n}^{0} \rightarrow \pi_{Y} \circ f^{0}=f \circ \pi_{X}$. Since our convergence is locally uniform, this implies $L\left(f_{n}^{0}\right)=f_{n} \rightarrow f$ $=L\left(f^{0}\right)$ and $L$ is a continuous function. Since $A(X, Y)^{0}$ is compact, $L$ is a homeomorphism. q.e.d.

\section{BiBLIOGRAPHY}

1. L. Ahlfors, Complex analysis, McGraw-Hill, New York, 1953.

2. L. Ahlfors and L. Sario, Riemann surfaces, Princeton Univ. Press, Princeton, N. J., 1960.

3. H. Behnke and K. Stein, Entwicklung analytischer Funktionen auf Riemannschen Flöchen, Math. Ann. 120 (1948), 430-461.

4. N. Bourbaki, Topologie générale, Chapter X, Actualités Sci. Ind. No. 1084, Hermann, Paris, 1949.

The Pennsylvania State University 